\title{
A NOTE ON HILBERT'S INEQUALITY
}

\author{
He Leping, Gao Mingzhe And Wei Shangrong
}

Abstract. In this paper, it is shown that some sharp results on Hilbert's inequality for double series can be obtained by means of the refined Cauchy's inequality.

Mathematics subject classification (2000): 26D15.

Key words and phrases: Double series, quadratic form, Hilbert's inequality, weight function.

\section{REFERENCES}

[1] G. H. Hardy, J. E. LitTlewood And G. Polya, Inequalities, Cambridge Univ. Press, Cambridge, 1995.

[2] Gao MingzHe, A Note on the Hardy-Hilbert Inequality, J. Math. Anal. Appl. Vol. 204, No. 1 (1996), 346-351.

[3] Gao Mingzhe And Yang Bichen, on the Extended Hilbert's Inequality, Proc. Amer. Math. Soc. Vol. 126, No. 3 (1998), 751-759.

[4] W. H. GReuB, Linear Algebra, Springer Verlag, Berlin, 1963, p. 161. 